\title{
The simplest wormhole in Rastall and k-essence theories
}

\author{
Kirill A. Bronnikov ${ }^{1,2,3, a}$, Vinícius A. G. Barcellos ${ }^{4}$, Laura P. de Carvalho ${ }^{4}$, Júlio C. Fabris ${ }^{3,4, b}$ \\ ${ }_{1}^{1}$ Center for Gravitation and Fundamental Metrology, VNIIMS, Ozyornaya ul. 46, Moscow 119361, Russia \\ ${ }^{2}$ Institute of Gravitation and Cosmology, Peoples' Friendship University of Russia RUDN University, ul. Miklukho-Maklaya 6, \\ Moscow 117198, Russia \\ ${ }^{3}$ National Research Nuclear University “MEPhI”, Kashirskoe sh. 31, Moscow 115409, Russia \\ ${ }^{4}$ Núcleo Cosmo-ufes and Departamento de Fíísica, CCE, Universidade Federal do Espírito Santo, Vitória, ES CEP 29075-910, Brazil
}

Received: 28 February 2021 / Accepted: 18 April 2021 / Published online: 6 May 2021

(C) The Author(s) 2021

\begin{abstract}
The geometry of the Ellis-Bronnikov wormhole is implemented in the Rastall and k-essence theories of gravity with a self-interacting scalar field. The form of the scalar field potential is determined in both cases. A stability analysis with respect to spherically symmetric time-dependent perturbations is carried out, and it shows that in k-essence theory the wormhole is unstable, like the original version of this geometry supported by a massless phantom scalar field in general relativity. In Rastall's theory, it turns out that a perturbative approach reveals the same inconsistency that was found previously for black hole solutions: time-dependent perturbations of the static configuration prove to be excluded by the equations of motion, and the wormhole is, in this sense, stable under spherical perturbations.
\end{abstract}

\section{Introduction}

Black holes and wormholes are remarkable predictions of the General Relativity theory (GR). The detection of gravitational waves emitted by merging of compact objects [1] and the recent image of a supermassive object at the center of the galaxy M87 [2] have brought black holes to the status of astrophysical objects whose existence in nature leaves little doubt. On the other hand, wormholes remain a hypothetical prediction of GR. In its simplest configuration, a wormhole is composed of two asymptotically flat Minkowskian spacetimes connected by a kind of tunnel. The two flat asymptotic regions are usually considered as different universes that are connected by a throat. One of the problematic aspects of wormhole configurations is the necessity of having negative energy, at least in the vicinity of the throat, in order that they could exist. Negative energy, which implies violation of

\footnotetext{
a e-mail: kb20@yandex.ru

b e-mail: julio.fabris@ cosmo-ufes.org (corresponding author)
}

the standard energy conditions, brings two main problems: the configuration can be unstable; or generally, the throat may not be traversable, in the sense that tidal forces may be huge, and possibly only pointlike objects may cross it from one universe to the other, except for some special cases. For a pedagogical description of wormhole properties, see Ref. [3].

The Ellis-Bronnikov (EB) wormhole $[4,5]$ is one of the simplest solutions of GR leading to a structure of two flat asymptotics connected by a throat. As a matter content, the EB wormhole solution uses a free massless scalar field with negative energy. Such field is normally denoted as a phantom scalar field. The configuration is, as could be expected, unstable due to the repulsive nature of the scalar field, see, e.g., [6,7] and references therein. Studies of static, spherically symmetric configurations in the presence of scalar fields have a long history, see [8,9] for the first seminal works on these lines. In parallel, there has been much effort to obtain wormhole solutions which, besides being traversable, would be stable and do not require exotic matter. However, it is hard to fulfill these requirements in the context of GR and even in its extensions for a simple reason: in order to cross the throat by coming from one region and arriving in the other, the geodesics must first converge and later diverge, and this property requires repulsive properties of matter which should thus violate at least some of the standard energy conditions. Still in the framework of GR there are, on the one hand, an example [10] of a stable wormhole supported by some kind of phantom matter, and, on the other hand, examples of phantom-free rotating cylindrically symmetric wormholes whose stability properties are yet unknown [11,12].

It is well known that a given metric may be a solution of the field equations of different theories of gravity or even in a single theory with different matter sources. An example is [10] where the EB wormhole in GR is supported by a particular kind of phantom perfect fluid instead of a scalar 
field as in $[4,5]$. In the case of different theories, the matter content should naturally depend on the theory under consideration. In this paper we explore the EB wormhole metric in two different theories. The first one is Rastall's theory of gravity [13] that abandons one of the cornerstones of GR, the usual conservation law for matter fields. The second one is the k-essence theory [14] which modifies the matter sector by introducing non-canonical forms for the kinetic term of a scalar field. The k-essence proposal may be connected with some fundamental theories inspired by quantum gravity. In both cases our goal is to verify if it is possible to avoid the usual difficulties in wormhole construction and to obtain stable solutions.

Previously, both Rastall and k-essence theories with a self-interacting scalar field have been studied in attempts to obtain static, spherically symmetric black hole solutions $[15,16]$. The solutions turned out to be quite exotic, mainly due to the asymptotic properties at infinity. A stability analysis has shown that those k-essence solutions were unstable [17]. However, surprisingly, the perturbation analysis of the Rastall solutions was shown to be inconsistent, and the stability issue remained unclear [18]. It has been speculated that this property of the Rastall solutions is connected with the absence of a Lagrangian formulation of this theory. A curious aspect of these k-essence and Rastall solutions is that they share some duality properties, in spite of quite different structures of the theories themselves [19].

Here we show that the EB wormhole metric can be a solution of both Rastall and k-essence theories under the condition that the potential describing the self-interaction of the scalar field is nonzero. We determine the form of this potential in each case. In the k-essence theory we use a powerlaw expression of the kinetic term, as in Ref. [16]. We perform a perturbation analysis of these solutions using a gaugeinvariant approach, and we find that the k-essence solution is unstable. Unlike that, in Rastall gravity the inconsistency found previously for black hole solutions re-appears here, and no time-dependent spherically symmetric perturbations can exist. Thus the EB metric in this framework may be said to be stable under such perturbations, but the existence of nonperturbative time-dependent solutions cannot be excluded, to say nothing of possible instabilities under less symmetric perturbations.

The paper is organized as follows. In Sect. 2 some general expression to be used in the calculations are settled out. In Sect. 3, the EB wormhole solution in GR is reproduced for comparison. In Sect. 4, the corresponding wormhole solution and the stability issue is presented for Rastall gravity. A similar analysis is carried out in k-essence theory in Sect. 5. In Sect. 6 we present our conclusions.

\section{General relations}

The goal of the present section is to give some general relations that will be used in the rest of the paper. We assume spherical symmetry but not necessarily staticity. This allows us to easily consider a static configuration which we will call the background and linear perturbations around it.

Spherical symmetry can be described by a metric of the form

$d s^{2}=e^{2 \gamma(t, x)} d t^{2}-e^{2 \alpha(t, x)} d x^{2}-e^{2 \beta(t, x)} d \Omega^{2}$,

where $d \Omega^{2}$ is the metric on a unit 2-sphere. If the configuration besides being spherically symmetric is also static, the metric coefficients $\alpha, \beta$ and $\gamma$ depend only on the radial coordinate $x$. There is freedom to reparametrize the radial coordinate, and its particular choice can be made by postulating a condition connecting the coefficients $\alpha, \beta$ and $\gamma$.

For the metric (1) the components of the Ricci tensor and expression for the d'Alambertian operator acting on a scalar field are given by

$$
\begin{aligned}
R_{0}^{0}= & e^{-2 \gamma}\left[\ddot{\alpha}+2 \ddot{\beta}+\dot{\alpha}^{2}+2 \dot{\beta}^{2}-\dot{\gamma}(\dot{\alpha}+2 \dot{\beta})\right] \\
& -e^{-2 \alpha}\left[\gamma^{\prime \prime}+\gamma^{\prime}\left(\gamma^{\prime}-\alpha^{\prime}+2 \beta^{\prime}\right)\right], \\
R_{1}^{1}= & e^{-2 \gamma}[\ddot{\alpha}+\dot{\alpha}(\dot{\alpha}-\dot{\gamma}+2 \dot{\beta})] \\
& -e^{-2 \alpha}\left[\gamma^{\prime \prime}+2 \beta^{\prime \prime}-\alpha^{\prime}\left(\gamma^{\prime}+2 \beta^{\prime}\right)+\gamma^{\prime 2}+2 \beta^{\prime 2}\right], \\
R_{2}^{2}= & e^{-2 \gamma}[\ddot{\beta}+\dot{\beta}(\dot{\alpha}-\dot{\gamma}+2 \dot{\beta})] \\
& -e^{-2 \alpha}\left[\beta^{\prime \prime}+\beta^{\prime}\left(\gamma^{\prime}-\alpha^{\prime}+2 \beta^{\prime}\right)\right]+e^{-2 \beta}, \\
R_{01}= & 2 \dot{\beta}^{\prime}+2\left(\beta^{\prime}-\gamma^{\prime}\right) \dot{\beta}-2 \beta^{\prime} \dot{\alpha}, \\
\square \phi= & e^{-2 \gamma}[\ddot{\phi}+(\dot{\alpha}-\dot{\gamma}+2 \dot{\beta}) \dot{\phi}] \\
& -e^{-2 \alpha}\left[\phi^{\prime \prime}+\left(\gamma^{\prime}-\alpha^{\prime}+2 \beta^{\prime}\right) \phi^{\prime}\right] .
\end{aligned}
$$

where dots denote $\partial / \partial t$ and primes $\partial / \partial x$.

In the case of a static space-time, all time derivatives disappear. However, in the study of small time-dependent perturbations around a given static solution at linear order, the linear terms with time derivatives become relevant.

In what follows we will discuss wormhole configurations in GR, Rastall's theory of gravity in the presence of a scalar field, and k-essence theories. In all these cases, the gravitational field equations can be written as the Einstein equations with appropriate stress-energy tensors $T_{\mu}^{\nu}$,

$R_{\mu}^{v}-\frac{1}{2} \delta_{\mu}^{v} R=-T_{\mu}^{v}$

or alternatively,

$R_{\mu}^{v}=-T_{\mu}^{v}+\frac{1}{2} \delta_{\mu}^{v} T_{\rho}^{\rho}$

where we are using units in which (in usual notations) $c=$ $8 \pi G=1$. These expressions are also valid in Rastall gravity, under a suitable redefinition of the stress-energy tensor. 


\section{Wormhole solution in GR with a free scalar field}

3.1 The (anti-)Fisher solution and the simplest wormhole

Let us begin with recalling a derivation of the EllisBronnikov wormhole solution in the context of GR. The equations in the presence of a free massless scalar field $\phi$ are given by

$$
\begin{aligned}
& R_{\mu}^{v}-\frac{1}{2} \delta_{\mu}^{v} R=-\epsilon\left(\phi_{; \mu} \phi^{; \nu}-\frac{1}{2} \delta_{\mu}^{v} \phi_{; \rho} \phi^{; \rho}\right), \\
& \square \phi=0,
\end{aligned}
$$

where the parameter $\epsilon$ indicates if the scalar field is of ordinary (canonical) $(\epsilon=1)$ or phantom $(\epsilon=-1)$ type. The Einstein equations rewritten in the form (5) read

$R_{\mu}^{\nu}=-\epsilon \phi_{; \mu} \phi^{; \nu}$.

Let us consider the static metric (1) and a scalar field $\phi=$ $\phi(x)$. The set of Eqs. (6) and (7) is then most conveniently solved using the harmonic coordinate condition $\alpha=2 \beta+\gamma$ [5] (under which we will denote the radial coordinate by $u$ ). Indeed, under this condition, the scalar field equation (7) and two independent equations among (8) (specifically, $R_{0}^{0}=0$ and $R_{0}^{0}+R_{2}^{2}=0$ ) take the form

$\phi^{\prime \prime}=0, \quad \gamma^{\prime \prime}=0, \quad \beta^{\prime \prime}+\gamma^{\prime \prime}=e^{2 \beta+2 \gamma}$,

(the prime stands here for $d / d u$ ). All of them are immediately integrated giving

$\phi=C u, \quad \gamma=-m u, \quad m, C=\mathrm{const}$,

(where two other integration constants are suppressed by choosing the scale of $t$ and the zero point of $\phi$ ), and

$$
\begin{aligned}
\left(\beta^{\prime}+\gamma^{\prime}\right)^{2} & =e^{2 \beta+2 \gamma}+k^{2} \operatorname{sign} k, \\
k & =\mathrm{const},
\end{aligned}
$$

where one more integration constant is suppressed by choosing the zero point of $u$. The solution of (11) depends on the sign of $k$ :

$e^{-\beta-\gamma}=k^{-1} \sinh (k u), k>0$,

$e^{-\beta-\gamma}=u, \quad k=0$,

$e^{-\beta-\gamma}=k^{-1} \sin (k u), \quad k<0$,

which can be jointly written as

$e^{-\beta-\gamma}=s(k, u) \equiv \begin{cases}k^{-1} \sinh (k u), & k>0, \\ u, & k=0, \\ k^{-1} \sin (k u), & k<0 .\end{cases}$

Lastly, substituting (10) and (11) into the $\left(\begin{array}{l}1 \\ 1\end{array}\right)$ component of Eq. (6) (which is an integral of other components), we obtain a relation between the integration constants:

$k^{2} \operatorname{sign} k=m^{2}+\frac{1}{2} \epsilon C^{2}$.
The metric takes the form

$$
\begin{aligned}
d s^{2}= & e^{-2 m u} d t^{2} \\
& -\frac{e^{2 m u}}{s^{2}(k, u)}\left(\frac{d u^{2}}{s^{2}(k, u)}+d \Omega^{2}\right),
\end{aligned}
$$

The constants $m$ and $C$ have the meaning of the Schwarzschild mass and the scalar charge, respectively. The coordinate $u$ is defined (without loss of generality) at $u>0$, and $u=0$ corresponds to spatial infinity (since there $\left.r(u) \equiv e^{\beta} \rightarrow \infty\right)$, at which the metric is asymptotically flat.

Equations (10), (14) and (15) give a joint representation of all static, spherically symmetric solutions to Eqs. (6), (7): Fisher's solution [8] of 1948 (repeatedly rediscovered afterwards) corresponding to $\epsilon=1$ (hence $k>0$ ) and all three branches of the solution for $\epsilon=-1$ [9] according to the signs of $k$ (sometimes called anti-Fisher solutions). Detailed descriptions of the corresponding geometries can be found, e.g., in $[5,6,20,21]$. Note that the instability of Fisher's solution under small radial perturbations was shown in [22], and that of anti-Fisher solutions in [6,7].

Our interest here is with wormhole solutions, which form the branch $\epsilon=-1, k<0$ : in this case, we have two flat spatial infinities at $u=0$ and $u=\pi /|k|$. The solution looks more transparent after the radial coordinate transformation

$x=b \cot (b u), \quad b:=|k|$,

which brings the solution to the form

$$
\begin{aligned}
d s^{2}= & e^{-2 m[\pi / 2-\arctan (x / b)]} d t^{2} \\
& -e^{2 m[\pi / 2-\arctan (x / b)]} d x^{2} \\
& -\left(x^{2}+b^{2}\right) d \Omega^{2}, \\
\phi= & C[\pi / 2-\arctan (x / b)],
\end{aligned}
$$

where $x$ is the so-called quasiglobal coordinate corresponding to the "gauge" $\alpha+\gamma=0$ in terms of the metric (1). The simplest configuration is obtained in the case of zero mass, $m=0$ :

$$
\begin{aligned}
d s^{2} & =d t^{2}-d x^{2}-\left(x^{2}+b^{2}\right) d \Omega^{2} \\
\phi & = \pm b \sqrt{2}[\pi / 2-\arctan (x / b)] .
\end{aligned}
$$

It is this solution that is called the Ellis wormhole [4], or the Ellis-Bronnikov (EB) wormhole, since this and more general scalar-vacuum and scalar-electrovacuum configurations were obtained and discussed in [5]. In terms of the metric (1), we have in (19)

$$
\begin{aligned}
& \alpha \equiv \gamma \equiv 0, \\
& \beta \equiv \log r(x)=\frac{1}{2} \log \left(x^{2}+b^{2}\right) .
\end{aligned}
$$




\subsection{Ellis wormhole instability in GR}

Consider now linear time-dependent spherically symmetric perturbations of the EB wormhole, described by additions $\delta \alpha, \delta \beta, \delta \gamma$ and $\delta \phi$ of the corresponding static (background) quantities, characterized by some smallness parameter $\varepsilon$. Following $[6,21,22]$, we choose the perturbation gauge $\delta \beta=0$. Then the perturbation equations following from (7) and (6) in the order $O(\varepsilon)$ can be written as

$$
\begin{aligned}
& e^{2(\alpha-\gamma)} \delta \ddot{\phi}-\delta \phi^{\prime \prime}-\left[2 \beta^{\prime}+\gamma^{\prime}-\alpha^{\prime}\right] \delta \phi^{\prime} \\
& -\left[\delta \gamma^{\prime}-\delta \alpha^{\prime}\right] \phi^{\prime}=0 . \\
& e^{2(\alpha-\gamma)} \delta \ddot{\alpha}-\delta \gamma^{\prime \prime} \\
& -\delta \gamma^{\prime}\left(2 \gamma^{\prime}-\alpha^{\prime}+2 \beta^{\prime}\right)+\gamma^{\prime} \delta \alpha^{\prime}=0, \\
& e^{2(\alpha-\gamma)} \delta \ddot{\alpha}-\delta \gamma^{\prime \prime}+\delta \alpha^{\prime}\left(\gamma^{\prime}+2 \beta^{\prime}\right) \\
& +\left(\alpha^{\prime}-2 \gamma^{\prime}\right) \delta \gamma^{\prime}=2 \epsilon \phi^{\prime} \delta \phi^{\prime}, \\
& \beta^{\prime}\left(\delta \gamma^{\prime}-\delta \alpha^{\prime}\right) \\
& -2 e^{2(\alpha-\beta)} \delta \alpha=0, \\
& -\beta^{\prime} \delta \dot{\alpha}=-\frac{\epsilon}{2} \phi^{\prime} \delta \dot{\phi} .
\end{aligned}
$$

These equations are written with an arbitrary radial coordinate $x$ in the background static metric but with a particular choice $(\delta \beta=0)$ of the perturbation gauge fixing the reference frame in perturbed space-time. We see that Eq. (26) can be integrated in $t$ giving

$$
\begin{aligned}
\delta \alpha & =-\frac{\eta}{2} \delta \phi+\xi(x), \\
\eta & =\frac{\phi^{\prime}}{\beta^{\prime}}
\end{aligned}
$$

with an arbitrary function $\xi(x)$; we will put $\xi(x) \equiv 0$ since only time-dependent perturbations are of interest.

For the Ellis wormhole solution (19), (20), such that $\gamma=$ $\alpha=0$ and $\epsilon=-1$, the remaining equations read

$$
\begin{aligned}
& \delta \ddot{\phi}-\delta \phi^{\prime \prime}-2 \beta^{\prime} \delta \phi^{\prime}-\phi^{\prime}\left(\delta \gamma^{\prime}-\delta \alpha^{\prime}\right)=0 . \\
& \delta \ddot{\alpha}-\delta \gamma^{\prime \prime}-2 \beta^{\prime} \delta \gamma^{\prime}=0, \\
& \delta \ddot{\alpha}-\delta \gamma^{\prime \prime}+2 \beta^{\prime} \delta \alpha^{\prime}=-2 \phi^{\prime} \delta \phi^{\prime}, \\
& \beta^{\prime}\left(\delta \gamma^{\prime}-\delta \alpha^{\prime}\right)-2 e^{-2 \beta} \delta \alpha=0,
\end{aligned}
$$

Subtracting equations (29) and (30) and using (27), we obtain

$\delta \gamma^{\prime}=\frac{1}{2}\left(\eta^{\prime} \delta \phi-\eta \delta \phi^{\prime}\right)$

Knowing $\delta \alpha$ and $\delta \gamma^{\prime}$, or equivalently using directly (31), we can eliminate the metric perturbations from the scalar field equation, which results in the following master equation:

$$
\delta \ddot{\phi}-\delta \phi^{\prime \prime}-2 \beta^{\prime} \delta \phi^{\prime}-\eta^{\prime} \phi^{\prime} \delta \phi=0 .
$$

Assuming the time dependence of $\delta \phi$ as a single spectral mode, $\delta \phi \propto e^{i \omega t}$,

$\delta \phi^{\prime \prime}+2 \beta^{\prime} \delta \phi^{\prime}+\left(\omega^{2}+\eta^{\prime} \phi^{\prime}\right) \delta \phi=0$.

Eliminating $\delta \phi^{\prime}$ by the substitution $\delta \phi=e^{-\beta} y(x)$, we arrive at the Schrödinger-like equation

$y^{\prime \prime}+\left\{\omega^{2}+\eta^{\prime} \phi^{\prime}-\beta^{\prime \prime}-\beta^{\prime 2}\right\} y=0$,

which coincides with the master equation found in [6] in the special case where $\alpha=\gamma=0$ and no scalar field potential is present. Using our expressions for $\phi$ and $\beta$ in the Ellis wormhole solution, we find

$y^{\prime \prime}+\left\{\omega^{2}-\left[\frac{b^{2}\left(3 x^{2}+2 b^{2}\right)}{x^{2}\left(x^{2}+b^{2}\right)^{2}}\right]\right\} y=0$.

The stability analysis requires imposing boundary condition. In our case, for $x \rightarrow \pm \infty$ it is reasonable to require $\delta \phi \rightarrow 0$, or $y=o(|x|)$. We can note that, asymptotically, Eq. (36)) has solutions in terms of Bessel functions,

$y(x)=A_{ \pm \sqrt{|x|}} J_{ \pm v}(\omega|x|), \quad v=\sqrt{3 b^{2}+1 / 4}, \quad A_{ \pm}=$const.

If $\omega=i \bar{\omega}$ (an imaginary frequency describing an instability), the solutions become

$$
\begin{aligned}
y(x)= & \sqrt{|x|}\left[A_{1} K_{v}(\bar{\omega}|x|)+A_{2} I_{v}(\bar{\omega}|x|)\right], \\
& A_{1,2}=\text { const. }
\end{aligned}
$$

where $K_{v}$ and $I_{v}$ are modified Bessel functions. The function $K_{v}$ tends to zero at large $|x|$, therefore, correct boundary conditions with imaginary $\omega$ are compatible with an instability. On the other hand, the positive nature of the effective potential $V_{\text {eff }}(x)$ in Eq. (36) (the expression in brackets) seems to exclude "energy levels" $\omega^{2}<0$. However, this argument cannot be directly applied because of a pole of this effective potential near the wormhole throat $x=0, V_{\text {eff }} \approx 2 / x^{2}$. This potential can be regularized by the appropriate Darboux transformation as described in [6,7]. The regularized potential turns out to contain a sufficiently deep well leading to the existence of an unstable perturbation mode, related to an evolving throat radius. The same result was previously obtained by a numerical study [23] which proved that an Ellis wormhole can either collapse to a black hole or inflate, depending on the sign of the initial perturbation.

The gauge condition we are using, $\delta \beta=0$, seems to prevent considering perturbations connected with a changing throat radius. But a more thorough investigation shows [6,7] that the unknown $\delta \phi$ in the master equation Eq. (33) is actually a gauge-invariant quantity. Indeed, a perturbation gauge may be described as a small coordinate shift $x^{\mu} \rightarrow x^{\mu}+\xi^{\mu}$ 
with $\xi^{\mu}=O(\varepsilon)$, or, in the $(x, t)$ subspace,

$t=\bar{t}+\xi^{0}(x, t)$,

$x=\bar{x}+\xi^{1}(x, t)$.

Then it can be directly verified that quantities like $\beta^{\prime} \delta \phi-\phi^{\prime} \delta \beta$ do not change under such coordinate shifts and are thus gauge-invariant, as well as their products with any background quantities, for example, $1 / \beta^{\prime}$. It follows that $\delta \phi$ in our consideration is the specific form of the gauge-invariant quantity $\psi=\delta \phi-\phi^{\prime} \delta \beta^{\prime} / \beta^{\prime}$ in the gauge $\delta \beta=0$. Other functions involved in (33) are combinations of the background quantities, therefore we can safely replace there $\delta \phi$ with $\psi$ and conclude that the whole master equation is gaugeinvariant. It can thus be used for considering any perturbations, including those with an evolving throat radius.

The gauge invariance issue is presented in more detail in $[6,7,21]$, and its analogue for perturbations in cosmology is discussed in [24]. In our further consideration we obtain gauge-invariant master equations for spherical perturbations in a similar way.

\section{Wormholes in Rastall gravity}

In Rastall's theory, if the source of gravity is a scalar field $\phi$ with a self-interaction potential $V(\phi)$, the field equations can be written as $[15,18]$

$$
\begin{aligned}
& R_{\mu}^{v}-\frac{1}{2} \delta_{\mu}^{v} R=-\epsilon\left\{\phi_{; \mu} \phi^{; v}+\frac{2-a}{2} \delta_{\mu}^{v} \phi^{; \rho} \phi ; \rho\right\} \\
& -(3-2 a) \delta_{\mu}^{v} V(\phi), \\
& \square \phi+(a-1) \frac{\phi^{; \rho} \phi^{; \sigma} \phi_{; \rho ; \sigma}}{\phi_{; \alpha} \phi^{; \alpha}} \\
& =-\epsilon(3-2 a) V_{\phi},
\end{aligned}
$$

where $a$ is a constant parameter of the theory, and at its special value $a=1$ we return to GR. Thus the effective stress-energy tensor of the scalar field reads

$T_{\mu}^{v}=\epsilon\left\{\phi_{; \mu} \phi^{; \nu}-\frac{2-a}{2} \delta_{\mu}^{v} \phi^{; \rho} \phi_{; \rho}\right\}+\delta_{\mu}^{v} W(\phi)$,

where $W(\phi)=(3-2 a) V(\phi)$. The modified Einstein equations can be rewritten as

$R_{\mu \nu}=-\epsilon\left\{\phi_{; \mu} \phi_{; \nu}+\frac{1-a}{2} g_{\mu \nu} \phi^{; \rho} \phi_{; \rho}\right\}+g_{\mu \nu} W(\phi)$.

For the static metric (1) and $\phi=\phi(x)$, the Rastall equations reduce to

$$
\begin{aligned}
& a \phi^{\prime \prime}+\left[\gamma^{\prime}-a \alpha^{\prime}+2 \beta^{\prime}\right] \phi^{\prime} \\
& =\epsilon e^{2 \alpha} W_{\phi}, \\
& \gamma^{\prime \prime}+\gamma^{\prime}\left(\gamma^{\prime}-\alpha^{\prime}+2 \beta^{\prime}\right) \\
& =-\frac{\epsilon}{2}(1-a) \phi^{\prime 2}-e^{2 \alpha} W,
\end{aligned}
$$

$$
\begin{aligned}
\gamma^{\prime \prime} & +2 \beta^{\prime \prime}-\alpha^{\prime}\left(\gamma^{\prime}+2 \beta^{\prime}\right)+\gamma^{\prime 2}+2 \beta^{2} \\
& =-\frac{\epsilon}{2}(3-a) \phi^{\prime 2}-e^{2 \alpha} W, \\
\beta^{\prime \prime} & +\beta^{\prime}\left(\gamma^{\prime}-\alpha^{\prime}+2 \beta^{\prime}\right)-e^{2(\alpha-\beta)} \\
& =-\frac{\epsilon}{2}(1-a) \phi^{\prime 2}-e^{2 \alpha} W,
\end{aligned}
$$

where $W_{\phi}=d W / d \phi$.

These equations become identical to the GR equations with a massless scalar field if we put

$\frac{\epsilon}{2}(1-a) \phi^{\prime 2}=-e^{2 \alpha} W$.

where we should take into account that $W_{\phi}=W^{\prime} / \phi^{\prime}$. Then all solutions for $\alpha, \beta, \gamma$ and $\phi^{\prime}$ are the same as in GR. But, a new element in Rastall gravity is that one needs a nonzero potential in order to create these solutions. For any given special solution, the potential can be determined from Eq. (47) or from any of the Eqs. (44)-(46).

In particular, for the Ellis wormhole solution (19), (20) the potential is found to be

$$
\begin{aligned}
& W(\phi) \equiv(3-2 a) V(\phi) \\
& =\frac{b^{2}(1-a)}{\left(x^{2}+b^{2}\right)^{2}} \\
& =\frac{1-a}{b^{2}} \cos ^{4}(\phi / \sqrt{2}) .
\end{aligned}
$$

Thus we have the simplest Ellis wormhole solution in Rastall gravity for any value of the Rastall parameter $a$.

\subsection{Wormhole stability in Rastall gravity}

To obtain the linear perturbation equations, we are consider Eqs. (40) and (42) using the expressions (2) for the Ricci tensor components, the gauge $\delta \beta=0$ and the potential (48) as a function of $\phi$. The equations read

$$
\begin{aligned}
& e^{2(\alpha-\gamma)} \delta \ddot{\phi}-a \delta \phi^{\prime \prime}-\left(\gamma^{\prime}-a \alpha^{\prime}+2 \beta^{\prime}\right) \delta \phi^{\prime}-\phi^{\prime}\left(\delta \gamma^{\prime}-a \delta \alpha^{\prime}\right) \\
& \quad=-\epsilon e^{2 \alpha}\left(2 W_{\phi} \delta \alpha+W_{\phi \phi} \delta \phi\right) \\
& e^{2(\alpha-\gamma)} \delta \ddot{\alpha}-\delta \gamma^{\prime \prime}-\left(2 \gamma^{\prime}-\alpha^{\prime}+2 \beta^{\prime}\right) \delta \gamma^{\prime}+\gamma^{\prime} \delta \alpha^{\prime} \\
& \quad=\epsilon(1-a) \phi^{\prime} \delta \phi^{\prime}+e^{2 \alpha}\left(2 W \delta \alpha+W_{\phi} \delta \phi\right) \\
& e^{2(\alpha-\gamma)} \delta \ddot{\alpha}-\delta \gamma^{\prime \prime}+\left(\gamma^{\prime}+2 \beta^{\prime}\right) \delta \alpha^{\prime}+\left(\alpha^{\prime}-2 \gamma^{\prime}\right) \delta \gamma^{\prime} \\
& =\epsilon(3-a) \phi^{\prime} \delta \phi^{\prime}+e^{2 \alpha}\left(2 W \delta \alpha+W_{\phi} \delta \phi\right) \\
& \beta^{\prime}\left(\delta \gamma^{\prime}-\delta \alpha^{\prime}\right)-2 e^{2(\alpha-\beta)} \delta \alpha \\
& =-\epsilon(1-a) \phi^{\prime} \delta \phi^{\prime}-e^{2 \alpha}\left(2 W \delta \alpha+W_{\phi} \delta \phi\right) \\
& -\beta^{\prime} \delta \dot{\alpha} \\
& =-\frac{\epsilon}{2} \phi^{\prime} \delta \dot{\phi}
\end{aligned}
$$

For our simplest case $\gamma=\alpha=0, \epsilon=-1$, the equations read

$$
\begin{aligned}
& \delta \ddot{\phi}-a \delta \phi^{\prime \prime}-2 \beta^{\prime} \delta \phi^{\prime}-\phi^{\prime}\left(\delta \gamma^{\prime}-a \delta \alpha^{\prime}\right) \\
& =-\epsilon\left(2 W_{\phi} \delta \alpha+W_{\phi \phi} \delta \phi\right),
\end{aligned}
$$




$$
\begin{aligned}
& \delta \ddot{\alpha}-\delta \gamma^{\prime \prime}+2 \beta^{\prime} \delta \gamma^{\prime} \\
& =\epsilon(1-a) \phi^{\prime} \delta \phi^{\prime}+\left(2 W \delta \alpha+W_{\phi} \delta \phi\right), \\
& \delta \ddot{\alpha}-\delta \gamma^{\prime \prime}-2 \beta^{\prime} \delta \alpha^{\prime} \\
& =\epsilon(3-a) \phi^{\prime} \delta \phi^{\prime}+\left(2 W \delta \alpha+W_{\phi} \delta \phi\right), \\
& \beta^{\prime}\left(\delta \gamma^{\prime}-\delta \alpha^{\prime}\right)-2 e^{-2 \beta} \delta \alpha \\
& =-\epsilon(1-a) \phi^{\prime} \delta \phi^{\prime}-\left(2 W \delta \alpha+W_{\phi} \delta \phi\right), \\
& \beta^{\prime} \delta \dot{\alpha}=-\frac{1}{2} \phi^{\prime} \delta \dot{\phi} .
\end{aligned}
$$

In [18] it has been shown that the stability problem for the Rastall theory in static, spherically symmetric configurations is inconsistent unless all perturbations are zero. It turns out that here we come across the same problem, as could be expected in view of those results. Indeed, from Eq. (58) we obtain, as previously in GR,

$\delta \alpha=-\frac{1}{2} \eta \delta \phi, \quad \eta=\frac{\phi^{\prime}}{\beta^{\prime}}$.

From the difference of (55) and (56) we obtain

$\delta \gamma^{\prime}=\frac{\epsilon}{2}\left(\eta \delta \phi^{\prime}-\eta^{\prime} \delta \phi\right)$.

On the other hand, from Eqs. (57) and (59) it follows

$$
\begin{aligned}
\delta \gamma^{\prime}= & -\frac{1}{2}\left(\eta \delta \phi^{\prime}-\eta^{\prime} \delta \phi\right) \\
& +(1-a) \eta\left[\delta \phi^{\prime}+\left(\frac{\eta}{4} \phi^{\prime}+\frac{\phi^{\prime \prime}}{\beta^{\prime}}\right) \delta \phi\right] .
\end{aligned}
$$

In this expression we have separated the terms contained in (59) from the others.

The expressions (59) and (61) coincide only if $a=1$, that is, when the Rastall theory reduces to GR, or if the quantity in brackets in (61) vanishes. In the second case, we can find explicitly the behavior of $\delta \phi$ :

$$
\begin{gathered}
\delta \phi=\phi_{1}(t) \exp \left(-\frac{3}{2} x+2 b \arctan \frac{x}{b}\right), \\
\phi_{1}(t)=\text { arbitrary function. }
\end{gathered}
$$

It is easy to see that, according to Eqs. (54) and (59), in the solution (62) the only possibility is $\phi_{1}(t)=0$. Hence, there is no perturbation at linear level, the same result as in [18]. Quite similarly to [18], it implies the absence of perturbations in all orders of smallness.

\section{Wormholes in k-essence theories}

\subsection{Static wormholes}

Let us consider the theory defined by the Lagrangian density

$\mathcal{L}=\sqrt{-g}[R+f(X)-2 V(\phi)]$, with the definitions

$X=\eta \phi_{; \rho} \phi^{; \rho}, \quad \eta= \pm 1$.

The scalar field equation has the form

$\eta f_{X} \square \phi+2 f_{X X} \phi^{, \rho} \phi^{, \sigma} \phi_{; \rho ; \sigma}=V_{\phi}$,

where the subscripts $X$ and $\phi$ denote derivatives with respect to the corresponding variables. The Einstein equations have the form (4) with the stress-energy tensor

$T_{\mu}^{v}=f_{X} \eta \phi_{, \mu} \phi^{, v}-\frac{1}{2} \delta_{\mu}^{\nu} f+\delta_{\mu}^{v} V$.

In the form (5) they can be written as

$R_{\mu}^{v}=\eta f_{X} \phi_{, \mu} \phi^{, v}-\frac{1}{2} \delta_{\mu}^{v}\left(-f+X f_{X}+2 V\right)$.

Let us now consider static, spherically symmetric spacetimes with the metric (1) and $\phi=\phi(x)$ and choose

$$
\begin{gathered}
f(X)=\epsilon f_{0} X^{n}, \quad n>0, \\
f_{0}>0, \quad \epsilon= \pm 1 .
\end{gathered}
$$

To avoid the possibility of complex values of $f(X)$, we must then fix $\eta=-1$, so that

$X=e^{-2 \alpha} \phi^{\prime 2}$.

The resulting equations of motion are

$$
\begin{aligned}
& n f_{0} e^{-2 n \alpha} \phi^{\prime 2 n-2} \\
& \quad \times\left\{(2 n-1) \phi^{\prime \prime}+\left[2 \beta^{\prime}+\gamma^{\prime}-(2 n-1) \alpha^{\prime}\right] \phi^{\prime}\right\}=-\epsilon V_{\phi}, \\
& \gamma^{\prime \prime}+\gamma^{\prime}\left(2 \beta^{\prime}+\gamma^{\prime}-\alpha^{\prime}\right) \\
& \quad=-\frac{\epsilon f_{0}}{2}(n-1) e^{2(1-n) \alpha} \phi^{\prime 2 n}-e^{2 \alpha} V, \\
& \gamma^{\prime \prime}+2 \beta^{\prime \prime}-\alpha^{\prime}\left(\gamma^{\prime}+2 \beta^{\prime}\right)+\gamma^{\prime 2}+2 \beta^{\prime 2} \\
& \quad=\frac{f_{0}}{2}(n+1) e^{2(1-n) \alpha} \phi^{\prime 2 n}-e^{2 \alpha} V, \\
& \beta^{\prime \prime}+\beta^{\prime}\left(2 \beta^{\prime}+\gamma^{\prime}-\alpha^{\prime}\right)-e^{2(\alpha-\beta)} \\
& =-\frac{\epsilon f_{0}}{2}(n-1) e^{2(1-n) \alpha} \phi^{\prime 2 n}-e^{2 \alpha} V .
\end{aligned}
$$

If we assume that the Ellis wormhole is a solution to Eqs. (70)-(73), we substitute there the expressions (21) and find that the sum and difference of (71) and (72) lead to the relations

$$
\begin{aligned}
V & =\frac{\epsilon f_{0}}{2}(n-1) e^{2 n \gamma} \phi^{\prime 2 n}, \\
n f_{0} \epsilon \phi^{2 n} & =\frac{2 r^{\prime \prime}}{r}=\frac{2 b^{2}}{\left(x^{2}+b^{2}\right)^{2}} .
\end{aligned}
$$

It follows that $\epsilon=-1$, which is natural for a wormhole solution that must violate the Null Energy Condition, so that $T_{0}^{0}-T_{1}^{1}<0$. As a result, we obtain the following explicit expressions for $\phi^{\prime}$ and the potential $V$ :

$\phi^{\prime}=C\left(x^{2}+b^{2}\right)^{-1 / n}$, 


$$
\begin{aligned}
C & =\left(\frac{2 b^{2}}{n f_{0}}\right)^{1 /(2 n)}, \\
V & =\frac{f_{0}}{2}(1-n) \phi^{\prime 2 n} \\
& =\frac{f_{0}}{2}(1-n) \frac{C^{2 n}}{\left(x^{2}+b^{2}\right)^{2}} .
\end{aligned}
$$

Substituting the expression for $\phi^{\prime}$ to (70) to find $V_{\phi}$, one can verify that the latter coincides with $V_{\phi}=V^{\prime} / \phi^{\prime}$ obtained directly from (76), thus confirming the correctness of the solution.

One can integrate $\phi^{\prime}$ given by (75) to obtain

$$
\begin{aligned}
\phi= & C x b^{-2 / n}{ }_{2} F_{1} \\
& \times\left(\frac{1}{2}, \frac{1}{n}, \frac{3}{2} ;-\frac{x^{2}}{b^{2}}\right)+\phi_{0},
\end{aligned}
$$

$\phi_{0}=$ const.

It is not simple to obtain a closed expression for $V(\phi)$ (to do that, we must invert the hypergeometric function). However, $V(\phi)$ is well defined since $\phi^{\prime}>0$ at all $x$. Also, at some special values of $n$ the hypergeometric function can reduce to simpler expressions.

Thus the Ellis wormhole solution is consistent with kessence theory with a potential.

\subsection{Instability of the k-essence solution}

The perturbation equations in the gauge $\delta \beta=0$, under the condition $\alpha=\gamma=0$ (but their perturbations are nonzero) can be written as

$$
\begin{aligned}
& \delta \ddot{\phi}-(2 n-1) \delta \phi^{\prime \prime}-2 \beta^{\prime} \delta \phi^{\prime}-\left\{\delta \gamma^{\prime}-(2 n-1) \delta \alpha^{\prime}\right\} \phi^{\prime} \\
&= \frac{1}{n f_{0}} \phi^{\prime(1-2 n)} \\
& \times\left\{V_{\phi \phi} \phi^{\prime} \delta \phi+V_{\phi}\left[2 n \phi^{\prime} \delta \alpha+2(1-n) \delta \phi^{\prime}\right]\right\} \\
& \delta \ddot{\alpha}- \delta \gamma^{\prime \prime}-2 \beta^{\prime} \delta \gamma^{\prime}=f_{0}(n-1) \phi^{\prime 2 n-1} \\
& \times\left[(1-n) \phi^{\prime} \delta \alpha+n \delta \phi^{\prime}\right]+\left(2 V \delta \alpha+V_{\phi} \delta \phi\right) \\
& \delta \ddot{\alpha}- {\left[\gamma^{\prime \prime}-2 \beta^{\prime} \delta \alpha^{\prime}=-f_{0}(n+1) \phi^{\prime 2 n-1}\right.} \\
& \times\left[(1-n) \phi^{\prime} \delta \alpha+n \delta \phi^{\prime}\right]+\left(2 V \delta \alpha+V_{\phi} \delta \phi\right) \\
& \beta^{\prime}\left(\delta \gamma^{\prime}-\delta \alpha^{\prime}\right)-2 e^{-2 \beta} \delta \alpha=f_{0}(1-n) \phi^{\prime 2 n-1} \\
& \quad \times\left[(1-n) \phi^{\prime} \delta \alpha+n \delta \phi^{\prime}\right]-\left(2 V \delta \alpha+V_{\phi} \delta \phi\right) \\
&-\beta^{\prime} \delta \dot{\alpha}=\frac{n}{2} f_{0} \phi^{\prime 2 n-1} \delta \dot{\phi}
\end{aligned}
$$

From Eq. (82) one obtains

$$
\delta \alpha=-\frac{n}{2} \bar{\eta} \delta \phi
$$

$$
\bar{\eta}=f_{0} \frac{\phi^{2 n-1}}{\beta^{\prime}} .
$$

Using this result, and combining Eqs. (79) and (80), we obtain

$$
\begin{aligned}
\delta \gamma^{\prime}= & \frac{n}{2}(1-2 n) \bar{\eta} \delta \phi^{\prime} \\
& +\frac{n}{2}\left[\bar{\eta}^{\prime}+(1-n) n \bar{\eta}^{2} \phi^{\prime}\right] \delta \phi
\end{aligned}
$$

This expression is consistent with (61) if $n=a=1$ and $\epsilon=-1$.

Using Eq. (81), the relation (83) and the background equations, we find again Eq. (84). Hence, unlike the Rastall case, the k-essence perturbation analysis is consistent, quite similarly to the results of $[17,18]$.

In addition, we can obtain an expression for $\delta \alpha^{\prime}$ by combining (81) with the difference of (79) and (80) as

$$
\begin{gathered}
\delta \alpha^{\prime}=\frac{1}{2 \beta^{\prime}}\left[-n f_{0} \phi^{\prime 2 n-1}-2 e^{-2 \beta} \delta \alpha\right. \\
\left.-2(1-n) f_{0} \beta^{\prime} \phi^{\prime 2 n-1} \delta \phi\right] .
\end{gathered}
$$

This expression coincides with the one obtained by directly differentiating (83), which verifies the correctness of the model and the calculations.

Now, to obtain the master equation for $\delta \phi$, we use the previous results and insert them into (78), along with the relations due to the background equations,

$$
\begin{aligned}
V_{\phi}= & -n \bar{\eta} \beta^{\prime}\left[(2 n-1) \frac{\phi^{\prime \prime}}{\phi^{\prime}}+2 \beta^{\prime}\right] \\
= & \frac{4 b^{2}}{C n}(n-1) x\left(b^{2}+x^{2}\right)^{-3+1 / n}, \\
V_{\phi \phi}= & -n\left\{4 \eta \beta^{\prime \prime} \frac{\beta^{\prime}}{\phi^{\prime}}+\bar{\eta}^{\prime} \frac{\beta^{\prime}}{\phi^{\prime}}\left[(2 n-1) \frac{\phi^{\prime \prime}}{\phi^{\prime}}+2 \beta^{\prime}\right]\right. \\
& \left.+(2 n-1) \bar{\eta}\left[\frac{\beta^{\prime \prime} \phi^{\prime \prime}}{\phi^{\prime 2}}+\beta^{\prime}\left(\frac{\phi^{\prime \prime \prime}}{\phi^{\prime 2}}-\frac{\phi^{\prime 2}}{\phi^{\prime 3}}\right)\right]\right\} \\
= & \frac{4 b^{2}(n-1)}{C^{2} n^{2}}\left(b^{2}+x^{2}\right)^{-4+2 / n} \\
& \times\left(n b^{2}+(2-5 n) x^{2}\right) .
\end{aligned}
$$

The final form of the master equation is

$$
\begin{aligned}
& -\delta \ddot{\phi}+(2 n-1) \delta \phi^{\prime \prime}+\left\{2 \beta^{\prime}+2 \frac{(1-n)}{n} \frac{V_{\phi}}{\bar{\eta} \beta^{\prime}}\right\} \delta \phi^{\prime} \\
& +\left\{n^{2} \bar{\eta}^{\prime} \phi^{\prime}+\frac{n(1-n)}{2} \bar{\eta}^{2} \phi^{\prime 2}-n \frac{\phi^{\prime}}{\beta^{\prime}} V_{\phi}+\frac{\phi^{\prime} V_{\phi \phi}}{n \bar{\eta} \beta^{\prime}}\right\} \delta \phi=0,
\end{aligned}
$$

or explicitly,

$$
\begin{aligned}
& \delta \ddot{\phi}-(2 n-1) \delta \phi^{\prime \prime}-2 \beta^{\prime}\left[1-\frac{2(n-1)^{2}}{n}\right] \delta \phi^{\prime} \\
& \quad+U(x) \delta \phi=0,
\end{aligned}
$$




$$
\begin{aligned}
& U(x)=\frac{2 b^{4}(2 n-1)}{x^{2}\left(x^{2}+b^{2}\right)^{2}}+\frac{2}{n^{2}\left(x^{2}+b^{2}\right)^{2}} \\
& \times\left[n b^{2}\left(n^{2}+n-1\right)-x^{2}\left(5 n^{2}-7 n+2\right)\right] .
\end{aligned}
$$

In general, the analysis of Eq. (89) is quite complicated. Let us begin with a simple example which shows a particular case where it is possible to explicitly prove the instability. Particularly, let us fix $n=1 / 2$. This case has been investigated in $[16,18]$ in search for black hole solutions and a study of their stability. In fact, there are some exotic types of black hole, but they are unstable. Now we are considering the same problem for the Ellis wormhole.

With $n=1 / 2$, Eq. (89) greatly simplifies and reads

$\delta \ddot{\phi}-\frac{2 x^{2}+b^{2}}{\left(x^{2}+b^{2}\right)^{2}} \delta \phi=0$,

which is easily integrated giving

$$
\begin{aligned}
\delta \phi & =K_{1}(x) e^{H(x) t}+K_{2}(x) e^{-H(x) t}, \\
H(x) & =\frac{\sqrt{2 x^{2}+b^{2}}}{x^{2}+b^{2}},
\end{aligned}
$$

where $K_{1}(x)$ and $K_{2}(x)$ are arbitrary functions. This evidently demonstrates the instability of the background configuration since the expression (91) exponentially grows with time if $K_{1} \neq 0$.

If $n=1$, we return to the situation in GR.

If $n<1 / 2$, Eq. (89) loses its hyperbolic nature, and the system is hydrodynamically unstable for the same reason as described in [17] and other papers.

Of more interest is the situation where $n>1 / 2$, in which Eq. (89) has a wave nature. It is then reasonable to get rid of the term containing $\delta \phi^{\prime}$ by putting

$$
\begin{aligned}
\delta \phi & =e^{-p \beta} y(x, t), \\
p & =\frac{2 n^{2}-5 n+2}{n(1-2 n)},
\end{aligned}
$$

after which the equation acquires the form

$\ddot{y}-(2 n-1) y^{\prime \prime}+\left[U(x)+(2 n-1)\left(\beta^{\prime \prime}+p \beta^{\prime 2}\right)\right] y=0$,

or, assuming a single spectral mode, $y \propto e^{i \omega t}$, so that $\ddot{y}=$ $-\omega^{2} y$

$$
\begin{aligned}
& y^{\prime \prime}+\left[\frac{\omega^{2}}{2 n-1}-V_{\mathrm{eff}}(x)\right] y=0, \\
& V_{\mathrm{eff}}(x)=\frac{U(x)}{2 n-1}+p \beta^{\prime \prime}+p^{2} \beta^{\prime 2} .
\end{aligned}
$$

It is the Schrödinger-like equation usually appearing in stability studies, for which the corresponding boundaryvalue problem should be solved in order to obtain stability conclusions. For perturbations of wormholes with phantom scalar fields, the effective potentials $V_{\text {eff }}(x)$ contain a singularity on the throat which can be regularized with proper Darboux transformations $[6,7,21]$ under the condition that $V_{\text {eff }}(x)=2 / x^{2}+O(1)$ near the throat (where $x$ is the "tortoise" coordinate in the wormhole space-time, and $x=0$ is the throat). This condition is generally satisfied for wormholes supported by phantom scalar fields with arbitrary potentials [21]. Surprisingly, this condition also holds for the effective potential $V_{\text {eff }}(x)$ in our equation (94) for wormholes in k-essence theory, so that the stability problem can be solved along the lines of $[6,7,21]$. This requires a separate study, to be performed in the near future.

\section{Conclusions and discussions}

The Ellis-Bronnikov solution represents the simplest analytical wormhole solution that can be obtained in GR. It consists of two asymptotically flat regions connected by a throat. This wormhole requires a massless, minimally coupled phantom scalar field: this means that all space, not only the throat, is filled with a field having negative energy density. In spite of being a very elegant and simple solution, the EB wormhole has a major drawback: it is unstable under linear perturbations. To look for a simple wormhole solution like the EB one that may not require phantom fields and/or that are stable is a challenge, even if some extensions of GR are employed.

It is well known that the same metric can be a solution of different gravitational theories. We have exploited this fact in order to investigate the conditions at which the EB wormhole metric can be a solution in the context of two extended gravity theories, Rastall gravity and k-essence. Rastall gravity is a more radical departure from GR since it modifies the usual expression for the conservation law. In some sense, Rastall gravity may be recast in the structure similar to GR where the expression for the energy-momentum tensor must be modified. Unlike that, k-essence is essentially a modification of the matter sector, keeping a Lagrangian formulation, by generalizing the usual kinetic expression. Both theories have applications, for example, in cosmology $[14,25,26]$ and black hole physics $[15,16]$.

We have shown that the EB metric can be a static, spherically symmetric solution in both Rastall and k-essence theories. To achieve that, a potential must be added in both cases, implying that, as opposed to GR, a self-interacting scalar field is required. The next step was to investigate the stability of these solutions in Rastall and k-essence cases. In Rastall gravity we face the same feature that was already found for black hole solutions: the usual perturbative approach leads to inconsistencies forcing to set all fluctuations near the background solution equal to zero. Perhaps this curious property is connected with the absence of a Lagrangian formulation. In k-essence theory, we have shown that the wormhole is 
unstable with respect to linear perturbation at least for the parameter $n$ in the range $n \leq 1 / 2$.

Some remarks must be added concerning these results of the perturbation analysis. First, only the simplest version of a wormhole metric has been investigated. This restriction is motivated by technical reasons since more complex configurations lead to very cumbersome expressions for the perturbations, even if a master equation can be obtained. Very probably, a numerical investigation can be necessary, which may imply new technical challenges. However, this remark mainly concerns the k-essence case. In Rastall gravity the situation may be more involved since we can expect that the inconsistency found here in the perturbative approach must remain, then a nonperturbative approach must be implemented. We hope to address these problems in future studies.

Finally, we remark that there has been quite a long discussion on the equivalence of GR and Rastall gravity. In fact, the number of degrees of freedom is the same in both cases, and this may allow for redefining the energy-momentum tensor to recast the Rastall equations in the same form as in GR. In the vacuum case, when the energy-momentum tensor is zero, the two theories simply coincide. However, we see that the situation changes if we consider particular forms of matter. Thus, in the present case, the self-interacting scalar field has the equation of motion different from that in GR, and so the change is not reduced to only redefining the energymomentum tensor. Thus, even though the number of degrees of freedom is the same in both theories, their coupling is different. The peculiar structure of this coupling in Rastall gravity manifests itself in stability study leading to the failure to apply a perturbation approach. Nevertheless, to be precise, we cannot exactly conclude that the Rastall wormhole configuration displayed here is stable since it is possible (even if much more difficult) to use a nonperturbative approach which may lead to nontrivial results on the stability of the solution.

Acknowledgements V.B., L.C. and J.F thank FAPES (Brazil) and $\mathrm{CNPq}$ (Brazil) for partial financial support. K.B. was supported by the RUDN University Strategic Academic Leadership Program. The research of K.B. was also funded by the Ministry of Science and Higher Education of the Russian Federation, Project "Fundamental properties of elementary particles and cosmology" N 0723-2020-0041, and by RFBR Project 19-02-00346.

Data Availability Statement This manuscript has no associated data or the data will not be deposited. [Authors' comment: The article has generated no data to be deposited.]

Open Access This article is licensed under a Creative Commons Attribution 4.0 International License, which permits use, sharing, adaptation, distribution and reproduction in any medium or format, as long as you give appropriate credit to the original author(s) and the source, provide a link to the Creative Commons licence, and indicate if changes were made. The images or other third party material in this article are included in the article's Creative Commons licence, unless indicated otherwise in a credit line to the material. If material is not included in the article's Creative Commons licence and your intended use is not permitted by statutory regulation or exceeds the permitted use, you will need to obtain permission directly from the copyright holder. To view a copy of this licence, visit http://creativecomm ons.org/licenses/by/4.0/.

Funded by SCOAP ${ }^{3}$.

\section{References}

1. B.P. Abbott et al., (LIGO Scientific Collaboration and Virgo Collaboration), Observation of gravitational waves from a binary black hole merger. Phys. Rev. Lett. 116, 061102 (2016)

2. The Event Horizon. Collaboration, The shadow of the supermassive black hole. Astrophys. J. Lett. 875, L1 (2019)

3. M.S. Morris, K.S. Thorne, Wormholes in space-time and their use for interstellar travel: A tool for teaching general relativity. Am. J. Phys. 56, 395 (1988)

4. H. Ellis, Ether flow through a drainhole: a particle model in general relativity. J. Math. Phys. 14, 104 (1973)

5. K.A. Bronnikov, Scalar-tensor theory and scalar charge. Acta Phys. Polon. B 4, 251 (1973)

6. K.A. Bronnikov, J.C. Fabris, A. Zhidenko, On the stability of scalarvacuum space-times. Eur. Phys. J. C 71, 1791 (2011)

7. J.A. Gonzalez, F.S. Guzman, O. Sarbach, Instability of wormholes supported by a ghost scalar field. I. Linear stability analysis. Class. Quantum Grav. 26, 015010 (2009)

8. I.Z. Fisher, Scalar mesostatic field with regard for gravitational effects. Zh. Eksp. Teor. Fiz. 18, 636 (1948). English translation: arXiv:gr-qc/9911008

9. O. Bergmann, R. Leipnik, Space-time structure of a static spherically symmetric scalar field. Phys. Rev. 107, 1157 (1957)

10. K.A. Bronnikov, L.N. Lipatova, I.D. Novikov, A.A. Shatskiy, Example of a stable wormhole in general relativity. Grav. Cosmol. 19, 269-274 (2013). arXiv:1312.6929

11. K.A. Bronnikov, V.G. Krechet,. Potentially observable cylindrical wormholes without exotic matter in GR. Phys. Rev. D 99, 084051 (2019). arXiv: 1807.03641

12. S.V. Bolokhov, K.A. Bronnikov, M.V. Skvortsova, Cylindrical wormholes: A search for viable phantom-free models in GR. Int. J. Mod. Phys. D 28, 1941008 (2019). arXiv:1903.09862

13. P. Rastall, Generalization of the Einstein theory. Phys. Rev. D 6, 3357 (1972)

14. C. Armendariz-Picon, T. Damour, V. Mukhanov, k-Inflation. Phys. Lett. B 458, 209 (1999)

15. K.A. Bronnikov, J.C. Fabris, O.F. Piattella, E.C. Santos, Static, spherically symmetric solutions with a scalar field in Rastall gravity. Gen. Rel. Grav. 48, 162 (2016)

16. K.A. Bronnikov, J.C. Fabris, D.C. Rodrigues, On horizons and wormholes in k-essence theories. Grav. Cosmol. 22, 26 (2016)

17. K.A. Bronnikov, J.C. Fabris, D.C. Rodrigues, On the instability of some k-essence space-times. Int. J. Mod. Phys. D 29, 2050016 (2020)

18. K.A. Bronnikov, J.C. Fabris, O.F. Piattella, D.C. Rodrigues, E.C.O. Santos, Rastall's theory of gravity: Spherically symmetric solutions and the stability problem. Gen. Rel. Grav. 53, 20 (2021). arXiv:2007.01945

19. K.A. Bronnikov, J.C. Fabris, O.F. Piattella, D.C. Rodrigues, E.C. Santos, Duality between k-essence and Rastall gravity. Eur. Phys. J. C 77, 409 (2017)

20. K.A. Bronnikov, S.G. Rubin, Black Holes, Cosmology, and Extra Dimensions (World Scientific, Singapore, 2013).

21. K.A. Bronnikov. Scalar fields as sources for wormholes and regular black holes. Particles 2018, 1, 5. arXiv:1802.00098 
22. K.A. Bronnikov, A.V. Khodunov, Scalar field and gravitational instability. Gen. Relat. Gravit. 11, 13 (1979)

23. H.-A. Shinkai, S.A. Hayward, Fate of the first traversible wormhole: black-hole collapse or inflationary expansion. Phys. Rev. D 66, 044005 (2002). arXiv:gr-qc/0205041

24. R.H. Brandenberger, H.A. Feldman, V.F. Mukhanov, Theory of cosmological perturbations. Phys. Rep. 215, 203 (1992)
25. C.E.M. Batista, M.H. Daouda, J.C. Fabris, O.F. Piattella, D.C. Rodrigues, Rastall cosmology and the $\Lambda$ CDM model. Phys. Rev. D 85, 084008 (2012)

26. J. Garriga, V.F. Mukhanov, Pertubations in k-inflation. Phys. Lett. B 458, 219 (1999) 